\title{
Vertex Numbers of Weighted Faces in Poisson Hyperplane Mosaics
}

\author{
Rolf Schneider
}

Received: 9 April 2009 / Accepted: 16 April 2010 / Published online: 8 May 2010

(C) Springer Science+Business Media, LLC 2010

\begin{abstract}
In the random mosaic generated by a stationary Poisson hyperplane process in $\mathbb{R}^{d}$, we consider the typical $k$-face weighted by the $j$-dimensional volume of the $j$-skeleton $(0 \leq j \leq k \leq d)$. We prove sharp lower and upper bounds for the expected number of its vertices.
\end{abstract}

Keywords Random tessellation · Poisson hyperplane mosaic - Weighted typical face $\cdot$ Expected vertex number $\cdot$ Volume product inequality

\section{Introduction}

Since the early work of Miles [5-7] and Matheron [2-4], stationary Poisson processes of hyperplanes and the tessellations induced by them have been an intensively studied topic of stochastic geometry. We refer to Chapter 10 of the book [9] for a description of later developments. The purpose of this note is an extension of a result (namely $[9,(10.52)])$ giving sharp estimates for the expected vertex number of the Poisson zero polytope. For stationary Poisson hyperplane mosaics, these inequalities are generalized from the zero cell, which can be viewed as the volume-weighted typical cell, to $L_{j}$-weighted typical $k$-faces $(0 \leq j \leq k \leq d)$. Here, $L_{j}(P)$ for a polytope $P$ is the sum of the $j$-volumes of its $j$-faces and thus the $j$-dimensional Hausdorff measure of its $j$-skeleton. The case $j=k$ was treated in [8]. For the general case, we shall have to extend a result of Favis and Weiss [1] on the distribution of $L_{j}$-weighted typical $d$-cells to lower-dimensional faces. After that, as in the previous work, the known inequalities for the volume product from convex geometry can be used, and they lead to extremal properties of either parallel mosaics or isotropic mosaics.

R. Schneider $(\varangle)$

Mathematisches Institut, Albert-Ludwigs-Universität, 79104, Freiburg i. Br., Germany

e-mail: rolf.schneider@math.uni-freiburg.de 


\section{Preliminaries}

We denote by $\mathbb{R}^{d}$ the $d$-dimensional Euclidean space with scalar product $\langle\cdot, \cdot\rangle$ and norm $\|\cdot\|$. The set $S^{d-1}:=\left\{u \in \mathbb{R}^{d}:\|u\|=1\right\}$ is its unit sphere. Lebesgue measure on $\mathbb{R}^{d}$ is denoted by $\lambda$, and $j$-dimensional Hausdorff measure by $\mathcal{H}^{j}$. In the following, measures and measurable functions on a topological space $T$ refer to its Borel $\sigma$-algebra, denoted by $\mathcal{B}(T)$. The Grassmannian $G(d, d-1)$ of $(d-1)$-dimensional linear subspaces of $\mathbb{R}^{d}$ and the affine Grassmannian $A(d, d-1)$ of hyperplanes in $\mathbb{R}^{d}$ are equipped with their usual topologies. We write hyperplanes in the form

$$
H(u, \tau)=\left\{x \in \mathbb{R}^{d}:\langle x, u\rangle=\tau\right\}=H(-u,-\tau)
$$

with $u \in S^{d-1}$ and $\tau \in \mathbb{R}$.

$\mathcal{K}$ is the space of convex bodies (nonempty compact convex sets) in $\mathbb{R}^{d}$, endowed with the topology induced by the Hausdorff metric, and $\mathcal{P}$ is the subspace of polytopes. For a polytope $P$, we denote by $\mathcal{F}_{j}(P)$ the set of its $j$-dimensional faces, and we write $\operatorname{skel}_{j} P:=\bigcup_{F \in \mathcal{F}_{j}(P)} F$ for the $j$-skeleton of $P(j=0, \ldots, \operatorname{dim} P)$.

We assume in the following some familiarity with the geometric processes (particles, flats, tessellations) considered in stochastic geometry (see [9], in particular, Chaps. 3, 4, 10). Throughout this note, $\widehat{X}$ is a stationary Poisson process of hyperplanes in $\mathbb{R}^{d}$, with a positive, locally finite intensity measure $\widehat{\Theta}$. The underlying probability space is $(\Omega, A, \mathbb{P})$, and mathematical expectation is denoted by $\mathbb{E}$. The intensity measure $\widehat{\Theta}$ has a decomposition

$$
\int_{A(d, d-1)} f \mathrm{~d} \widehat{\Theta}=\widehat{\gamma} \int_{S^{d-1}} \int_{-\infty}^{\infty} f(H(u, \tau)) \mathrm{d} \tau \widehat{\varphi}(\mathrm{d} u)
$$

for every nonnegative measurable function $f$ on $A(d, d-1)$ (see [9, Theorem 4.4.2 and (4.30)]). Here $\widehat{\gamma}>0$ is the intensity of $\widehat{X}$, and $\widehat{\varphi}$ is an even probability measure on the unit sphere $S^{d-1}$, the spherical directional distribution of $\widehat{X}$. We assume that $\widehat{\varphi}$ is not concentrated on a great subsphere of $S^{d-1}$.

Every realization of $\widehat{X}$ gives rise to a tessellation or mosaic, the cells of which are the closures of the connected components of the complement of the hyperplanes in $\widehat{X}$. The particle process $X$ of these cells is the Poisson hyperplane tessellation induced by $\widehat{X}$. For $k \in\{0, \ldots, d\}$, we denote by $X^{(k)}=\bigcup_{P \in X} \mathcal{F}_{k}(P)$ the particle process formed by the $k$-dimensional faces of all cells of $X$. The stationary particle process $X^{(k)}$ has a positive, locally finite intensity measure, denoted by $\Theta^{(k)}$. Again, there is a decomposition

$$
\int_{\mathcal{K}} f \mathrm{~d} \Theta^{(k)}=\gamma^{(k)} \int_{\mathcal{K}_{0}} \int_{\mathbb{R}^{d}} f(K+x) \lambda(\mathrm{d} x) \mathbb{Q}^{(k)}(\mathrm{d} K)
$$

for every nonnegative measurable function $f$ on $\mathcal{K}$ (see [9, (4.3)]). Here $\mathcal{K}_{0}$ is the space of convex bodies $K$ with $s(K)=0$, where $s$ denotes the Steiner point (which we use here as center function). The positive number $\gamma^{(k)}$ is the intensity of $X^{(k)}$, and the probability measure $\mathbb{Q}^{(k)}$ on $\mathcal{K}_{0}$ is the grain distribution of $X^{(k)}$. 


\section{Weighted Faces}

Average $k$-faces of the random tessellation $X$ can be defined in different but equally natural ways. The typical $k$-face of $X$ is, by definition, the random polytope $Z^{(k)}$ with distribution $\mathbb{Q}^{(k)}$. Intuitively (but only heuristically), it is obtained as follows. In a realization of $X$, we pick out at random one of the $k$-faces, with equal chances for every $k$-face (this, of course, makes only sense within a bounded region of space), and translate it to bring its Steiner point to the origin. If, instead, we make this random choice with chances proportional to some given translation-invariant positive measurable function $w$ defined on $k$-polytopes, we obtain the $w$-weighted typical $k$-face. Formally, one can define this as the random polytope $Z_{w}^{(k)}$ with distribution given by

$$
\begin{aligned}
\mathbb{P}\left(Z_{w}^{(k)} \in A\right) & =\frac{1}{\mathbb{E} w\left(Z^{(k)}\right)} \mathbb{E}\left[\mathbf{1}_{A}\left(Z^{(k)}\right) w\left(Z^{(k)}\right)\right] \\
& =\frac{1}{\mathbb{E} w\left(Z^{(k)}\right)} \int_{\mathcal{K}_{0}} \mathbf{1}_{A}(K) w(K) \mathbb{Q}^{(k)}(\mathrm{d} K)
\end{aligned}
$$

for $A \in \mathcal{B}(\mathcal{P})$. This definition comprises the intuitive idea of selecting, with weights, from large bounded regions, as can be seen from the relation

$$
\mathbb{P}\left(Z_{w}^{(k)} \in A\right)=\lim _{r \rightarrow \infty} \frac{\mathbb{E} \sum_{F \in X^{(k)}, F \subset r W} \mathbf{1}_{A}(F-s(F)) w(F)}{\mathbb{E} \sum_{F \in X^{(k)}, F \subset r W} w(F)} .
$$

It holds for $A \in \mathcal{B}(\mathcal{P})$ and any compact convex set $W \subset \mathbb{R}^{d}$ with $\lambda(W)>0$ and is a special case of [9, Theorem 4.1.3].

By the standard extension procedure from indicator functions to nonnegative measurable functions $f$, relation (3) yields

$$
\int_{\mathcal{K}_{0}} f(K) w(K) \mathbb{Q}^{(k)}(\mathrm{d} K)=\mathbb{E} w\left(Z^{(k)}\right) \mathbb{E} f\left(Z_{w}^{(k)}\right) .
$$

Here we are interested in the weighting function given by

$$
L_{j}(P)=\mathcal{H}^{j}\left(\operatorname{skel}_{j} P\right)=\sum_{F \in \mathcal{F}_{j}(P)} \mathcal{H}^{j}(F),
$$

the total $j$-dimensional volume of the $j$-faces of $P$ for $0 \leq j \leq \operatorname{dim} P$. In particular, if $P$ is a $d$-dimensional polytope, then $L_{d}(P)$ is the volume, $L_{d-1}(P)$ is the surface area, $L_{1}(P)$ is the total edge length, and $L_{0}(P)$ is the number of vertices of $P$. We denote by $Z_{k, j}$ the $L_{j}$-weighted typical $k$-face of $X$. Note that the weighted typical $k$-face $Z_{0}^{(k)}$ considered in [8] differs from $Z_{k, k}$ by a random translation.

We need a Slivnyak-type result for $Z_{k, j}$ (Theorem 1). For this, we define the measure $\phi_{k}$ on $G(d, d-1)^{k}$ by

$$
\phi_{k}(A):=\frac{\widehat{\gamma}^{k}}{k ! \widehat{\gamma}_{k}} \int_{\left(S^{d-1}\right)^{k}} \mathbf{1}_{A}\left(u_{1}^{\perp}, \ldots, u_{k}^{\perp}\right) \nabla_{k}\left(u_{1}, \ldots, u_{k}\right) \widehat{\varphi}^{k}\left(\mathrm{~d}\left(u_{1}, \ldots, u_{k}\right)\right)
$$

for $A \in \mathcal{B}\left(G(d, d-1)^{k}\right)$, where $u^{\perp}:=\left\{x \in \mathbb{R}^{d}:\langle x, u\rangle=0\right\}$, and $\nabla_{k}\left(u_{1}, \ldots, u_{k}\right)$ denotes the $k$-dimensional volume of the parallelepiped spanned by $u_{1}, \ldots, u_{k}$. The 
constant $\widehat{\gamma}_{k}$ is the intensity of the intersection process of order $k$ of the hyperplane process $\widehat{X}$. According to [9, Theorem 4.4.8], it is given by

$$
\widehat{\gamma}_{k}=\frac{\widehat{\gamma}^{k}}{k !} \int_{\left(S^{d-1}\right)^{k}} \nabla_{k}\left(u_{1}, \ldots, u_{k}\right) \widehat{\varphi}^{k}\left(\mathrm{~d}\left(u_{1}, \ldots, u_{k}\right)\right) .
$$

Thus, $\phi_{k}$ is a probability measure.

Let $h$ be a function on the space of $k$-dimensional polytopes. Let $\eta$ be a locally finite system of hyperplanes in $\mathbb{R}^{d}$ which are in general position (below, $\eta$ also has this meaning). "General position" means that every $m$-dimensional plane of $\mathbb{R}^{d}$ is contained in at most $d-m$ hyperplanes of $\eta$ for $m=0, \ldots, d$. (Note that for $\eta=$ $\widehat{X}(\omega), \omega \in \Omega$, general position can always be assumed, since for a stationary Poisson hyperplane process, this holds almost surely.) For $j \leq k$ and $x \in \mathbb{R}^{d}$, we define

$$
h_{k, j}(\eta, x):=h\left(K_{1}(x)\right)+\cdots+h\left(K_{n}(x)\right)
$$

if $x$ is in the relative interior of a $j$-face of the tessellation induced by $\eta$ and $K_{1}(x), \ldots, K_{n}(x)$ are the $k$-faces of $X$ containing that $j$-face, and we define $h_{k, j}(\eta, x):=0$ if $x$ is not in the relative interior of a $j$-face of the tessellation. The number $n$ is obtained as follows. The affine hull of a $j$-face is the intersection of precisely $d-j$ hyperplanes of $\eta$. Any $d-k$ of these hyperplanes generate (by intersection) a $k$-plane containing the $j$-face. Within this $k$-plane, there are $2^{k-j} k$-faces of the tessellation containing the $j$-face. Hence,

$$
n=2^{k-j}\left(\begin{array}{l}
d-j \\
d-k
\end{array}\right) .
$$

Theorem 1 Let $0 \leq j \leq k \leq d$. If $h$ is a translation-invariant nonnegative measurable function on $k$-polytopes, then

$$
\begin{aligned}
& \mathbb{E} h\left(Z_{k, j}\right) \\
& \quad=\frac{2^{j-k}}{\left(\begin{array}{c}
d-j \\
d-k
\end{array}\right)} \int_{G(d, d-1)^{k}} \mathbb{E} h_{k, j}\left(\widehat{X} \cup\left\{H_{1}, \ldots, H_{d-j}\right\}, 0\right) \phi_{d-j}\left(\mathrm{~d}\left(H_{1}, \ldots, H_{d-j}\right)\right) .
\end{aligned}
$$

For $k=d$, this formula was proved (with different notation and a partially different argument) by Favis and Weiss [1].

\section{Proof of Theorem 1}

Let $j, k$, and $h$ be as in Theorem 1 . Let $B \in \mathcal{B}\left(\mathbb{R}^{d}\right)$ be a set with $\lambda(B)=1$. With Campbell's theorem [9, Theorem 3.1.2] and decomposition (2) of the intensity measure $\Theta^{(k)}$ of $X^{(k)}$, we obtain

$$
\begin{aligned}
\mathbb{E} & \sum_{K \in X^{(k)}} h(K) \mathcal{H}^{j}\left(B \cap \operatorname{skel}_{j} K\right) \\
= & \int_{\mathcal{K}} h(K) \mathcal{H}^{j}\left(B \cap \operatorname{skel}_{j} K\right) \Theta^{(k)}(\mathrm{d} K)
\end{aligned}
$$




$$
\begin{aligned}
& =\gamma^{(k)} \int_{\mathcal{K}_{0}} \int_{\mathbb{R}^{d}} h(K+x) \mathcal{H}^{j}\left(B \cap \operatorname{skel}_{j}(K+x)\right) \lambda(\mathrm{d} x) \mathbb{Q}^{(k)}(\mathrm{d} K) \\
& =\gamma^{(k)} \int_{\mathcal{K}_{0}} h(K) \int_{\mathbb{R}^{d}} \mathcal{H}^{j}\left(B \cap\left(\operatorname{skel}_{j} K\right)+x\right) \lambda(\mathrm{d} x) \mathbb{Q}^{(k)}(\mathrm{d} K) .
\end{aligned}
$$

By Fubini's theorem (or see [9, Theorem 5.2.1]), for a polytope $K$,

$$
\int_{\mathbb{R}^{d}} \mathcal{H}^{j}\left(B \cap\left(\left(\operatorname{skel}_{j} K\right)+x\right)\right) \lambda(\mathrm{d} x)=\mathcal{H}^{j}\left(\operatorname{skel}_{j} K\right) \lambda(B)=L_{j}(K),
$$

and hence, using (4), we have

$$
\begin{aligned}
\mathbb{E} \sum_{K \in X^{(k)}} h(K) \mathcal{H}^{j}\left(B \cap \operatorname{skel}_{j} K\right) & =\gamma^{(k)} \int_{\mathcal{K}_{0}} h(K) L_{j}(K) \mathbb{Q}^{(k)}(\mathrm{d} K) \\
& =\gamma^{(k)} \mathbb{E} L_{j}\left(Z^{(k)}\right) \mathbb{E} h\left(Z_{k, j}\right) .
\end{aligned}
$$

In the following computations, we make use of some obvious facts. If $F \in X^{(j)}$ and $K \in X^{(k)}$, then for any point $x$ in the relative interior of $F$, the inclusion $F \subset K$ holds if and only if $x \in K$; hence,

$$
\mathbf{1}\{F \subset K\} \mathcal{H}^{j}(B \cap F)=\int_{B \cap F} \mathbf{1}_{K}(x) \mathcal{H}^{j}(\mathrm{~d} x) .
$$

Every $j$-face of a $k$-face of $X$ is a $j$-face of $X$ and is contained in the intersection of $d-j$ a.s. uniquely determined hyperplanes of $\widehat{X}$. Every such intersection is the union of $j$-faces of $X$ that have pairwise no common relatively interior points. This gives

$$
\begin{aligned}
\gamma^{(k)} & \mathbb{E} L_{j}\left(Z^{(k)}\right) \mathbb{E} h\left(Z_{k, j}\right) \\
& =\mathbb{E} \sum_{K \in X^{(k)}} h(K) \mathcal{H}^{j}\left(B \cap \operatorname{skel}_{j} K\right) \\
& =\mathbb{E} \sum_{K \in X^{(k)}} h(K) \sum_{F \in X^{(j)}} \mathbf{1}\{F \subset K\} \mathcal{H}^{j}(B \cap F) \\
& =\mathbb{E} \sum_{F \in X^{(j)}} \sum_{K \in X^{(k)}} h(K) \int_{B \cap F} \mathbf{1}_{K}(x) \mathcal{H}^{j}(\mathrm{~d} x) \\
& =\mathbb{E} \sum_{F \in X^{(j)}} \int_{B \cap F} h_{k, j}(\widehat{X}, x) \mathcal{H}^{j}(\mathrm{~d} x) \\
& =\frac{1}{(d-j) !} \mathbb{E} \sum_{\left(H_{1}, \ldots, H_{d-j}\right) \in \widehat{X}_{\neq}^{d-j}} \int_{B \cap H_{1} \cap \cdots \cap H_{d-j}} h_{k, j}(\widehat{X}, x) \mathcal{H}^{j}(\mathrm{~d} x),
\end{aligned}
$$


where $\eta_{\neq}^{d-j}:=\left\{\left(H_{1}, \ldots, H_{d-j}\right) \in \eta^{d-j}: H_{i}\right.$ pairwise distinct $\}$. We use the abbreviation

$$
\int_{B \cap H_{1} \cap \cdots \cap H_{d-j}} h_{k, j}(\widehat{X}, x) \mathcal{H}^{j}(\mathrm{~d} x)=: f\left(\widehat{X}, H_{1}, \ldots, H_{d-j}\right)
$$

and apply the Slivnyak-Mecke formula (see [9, Corollary 3.2.3]) with this function $f$. Employing decomposition (1) of the intensity measure $\widehat{\Theta}$, we get

$$
\begin{aligned}
\mathbb{E} & \sum_{\left(H_{1}, \ldots, H_{d-j}\right) \in \widehat{X}_{\neq}^{d-j}} f\left(\widehat{X}, H_{1}, \ldots, H_{d-j}\right) \\
= & \int_{A(d, d-1)^{d-j}} \mathbb{E} f\left(\widehat{X} \cup\left\{H_{1}, \ldots, H_{d-j}\right\}, H_{1}, \ldots, H_{d-j}\right) \\
& \times \widehat{\Theta}^{d-j}\left(\mathrm{~d}\left(H_{1}, \ldots, H_{d-j}\right)\right) \\
= & \widehat{\gamma}^{d-j} \int_{\left(S^{d-1}\right)^{d-j}} \int_{-\infty}^{\infty} \cdots \int_{-\infty}^{\infty} \mathbb{E} f\left(\widehat{X} \cup\left\{H\left(u_{1}, \tau_{1}\right), \ldots, H\left(u_{d-j}, \tau_{d-j}\right)\right\},\right. \\
& \left.H\left(u_{1}, \tau_{1}\right), \ldots, H\left(u_{d-j}, \tau_{d-j}\right)\right) \mathrm{d} \tau_{1} \cdots \mathrm{d} \tau_{d-j} \widehat{\varphi}^{d-j}\left(\mathrm{~d}\left(u_{1}, \ldots, u_{d-j}\right)\right) .
\end{aligned}
$$

Inserting this, we obtain

$$
\begin{aligned}
\mathbb{E} h\left(Z_{k, j}\right)= & \frac{\widehat{\gamma}^{d-j}}{(d-j) ! \gamma^{(k)} \mathbb{E} L_{j}\left(Z^{(k)}\right)} \\
& \times \int_{\left(S^{d-1}\right)^{d-j}} \int_{-\infty}^{\infty} \cdots \int_{-\infty}^{\infty} \mathbb{E} \int_{\substack{B \cap H\left(u_{1}, \tau_{1}\right) \cap \cdots \\
\cap H\left(u_{d-j}, \tau_{d-j}\right)}} h_{k, j}\left(\widehat { X } \cup \left\{H_{1}\left(u_{1}, \tau_{1}\right), \ldots,\right.\right. \\
& \left.\left.H\left(u_{d-j}, \tau_{d-j}\right)\right\}, x\right) \mathcal{H}^{j}(\mathrm{~d} x) \mathrm{d} \tau_{1} \cdots \mathrm{d} \tau_{d-j} \widehat{\varphi}^{d-j}\left(\mathrm{~d}\left(u_{1}, \ldots, u_{d-j}\right)\right) .
\end{aligned}
$$

For fixed linearly independent unit vectors $u_{1}, \ldots, u_{d-j}$ and real numbers $\tau_{1}, \ldots$, $\tau_{d-j}$, let $z=z\left(u_{1}, \ldots, u_{d-j}, \tau_{1}, \ldots, \tau_{d-j}\right)$ be the vector in $\operatorname{lin}\left\{u_{1}, \ldots, u_{d-j}\right\}$ with $\left\langle z, u_{i}\right\rangle=\tau_{i}$ for $i=1, \ldots, d-j$. In the following computations, we write $H\left(u_{i}, \tau_{i}\right)=$ $u_{i}^{\perp}+z$, substitute $x$ by $y+z$, and observe that $x \in u_{i}^{\perp}+z$, together with $x=y+z$, implies $u_{i}^{\perp}+z=u_{i}^{\perp}+y+z$. Since $h$ is translation invariant and $\widehat{X}$ is stationary, we get

$$
\begin{gathered}
\mathbb{E} \int_{\substack{B \cap H\left(u_{1}, \tau_{1}\right) \cap \ldots \\
\cap H\left(u_{d-j}, \tau_{d-j}\right)}} h_{k, j}\left(\widehat{X} \cup\left\{H_{1}\left(u_{1}, \tau_{1}\right), \ldots, H\left(u_{d-j}, \tau_{d-j}\right)\right\}, x\right) \mathcal{H}^{j}(\mathrm{~d} x) \\
=\mathbb{E} \int_{B \cap\left(u_{1}^{\perp}+z\right) \cap \cdots \cap\left(u_{d-j}^{\perp}+z\right)} h_{k, j}\left(\widehat{X} \cup\left\{u_{1}^{\perp}+z, \ldots, u_{d-j}^{\perp}+z\right\}, x\right) \mathcal{H}^{j}(\mathrm{~d} x) \\
=\int_{(B-z) \cap u_{1}^{\perp} \cap \cdots \cap u_{d-j}^{\perp}} \mathbb{E} h_{k, j}\left((\widehat{X}-y-z) \cup\left\{u_{1}^{\perp}, \ldots, u_{d-j}^{\perp}\right\}, 0\right) \mathcal{H}^{j}(\mathrm{~d} y) \\
=\mathbb{E} h_{k, j}\left(\widehat{X} \cup\left\{u_{1}^{\perp}, \ldots, u_{d-j}^{\perp}\right\}, 0\right) \mathcal{H}^{j}\left((B-z) \cap u_{1}^{\perp} \cap \cdots \cap u_{d-j}^{\perp}\right) .
\end{gathered}
$$


This yields

$$
\begin{aligned}
\mathbb{E} h\left(Z_{k, j}\right) & \\
= & \frac{\widehat{\gamma}^{d-j}}{(d-j) ! \gamma^{(k)} \mathbb{E} L_{j}\left(Z^{(k)}\right)} \\
& \times \int_{\left(S^{d-1}\right)^{d-j}} \int_{-\infty}^{\infty} \cdots \int_{-\infty}^{\infty} \mathcal{H}^{j}\left((B-z) \cap u_{1}^{\perp} \cap \cdots \cap u_{d-j}^{\perp}\right) \\
& \times \mathbb{E} h_{k, j}\left(\widehat{X} \cup\left\{u_{1}^{\perp}, \ldots, u_{d-j}^{\perp}\right\}, 0\right) \mathrm{d} \tau_{1} \cdots \mathrm{d} \tau_{d-j} \widehat{\varphi}^{d-j}\left(\mathrm{~d}\left(u_{1}, \ldots, u_{d-j}\right)\right) .
\end{aligned}
$$

Recalling how $z$ depends on $u_{1}, \ldots, u_{d-j}, \tau_{1}, \ldots, \tau_{d-j}$, we see from the transformation formula (13) in [8], writing $U:=\operatorname{lin}\left\{u_{1}, \ldots, u_{d-j}\right\}$, that

$$
\begin{gathered}
\int_{-\infty}^{\infty} \ldots \int_{-\infty}^{\infty} \mathcal{H}^{j}\left((B-z) \cap u_{1}^{\perp} \cap \cdots \cap u_{d-j}^{\perp}\right) \mathrm{d} \tau_{1} \cdots \mathrm{d} \tau_{d-j} \\
=\nabla_{d-j}\left(u_{1}, \ldots, u_{d-j}\right) \int_{U} \mathcal{H}^{j}\left(B \cap\left(U^{\perp}+z\right)\right) \mathcal{H}^{d-j}(\mathrm{~d} z) \\
=\nabla_{d-j}\left(u_{1}, \ldots, u_{d-j}\right) \lambda(B)=\nabla_{d-j}\left(u_{1}, \ldots, u_{d-j}\right) .
\end{gathered}
$$

Using definition (5), we conclude that

$\mathbb{E} h\left(Z_{k, j}\right)$

$$
\begin{aligned}
= & \frac{\widehat{\gamma}^{d-j}}{(d-j) ! \gamma^{(k)} \mathbb{E} L_{j}\left(Z^{(k)}\right)} \int_{\left(S^{d-1}\right)^{d-j}} \mathbb{E} h_{k, j}\left(\widehat{X} \cup\left\{u_{1}^{\perp}, \ldots, u_{d-j}^{\perp}\right\}, 0\right) \\
& \times \nabla_{d-j}\left(u_{1}, \ldots, u_{d-j}\right) \widehat{\varphi}^{d-j}\left(\mathrm{~d}\left(u_{1}, \ldots, u_{d-j}\right)\right) \\
= & \frac{\widehat{\gamma}_{d-j}}{\gamma^{(k)}} \frac{1}{\mathbb{E} L_{j}\left(Z^{(k)}\right)} \int_{G(d, d-1)^{d-j}} \mathbb{E} h_{k, j}\left(\widehat{X} \cup\left\{H_{1}, \ldots, H_{d-j}\right\}, 0\right) \\
& \times \phi_{d-j}\left(\mathrm{~d}\left(H_{1}, \ldots, H_{d-j}\right)\right) .
\end{aligned}
$$

With $h=1$ and using (6), this gives

$$
1=\frac{\widehat{\gamma}_{d-j}}{\gamma^{(k)}} \frac{1}{\mathbb{E} L_{j}\left(Z^{(k)}\right)} \cdot 2^{k-j}\left(\begin{array}{l}
d-j \\
d-k
\end{array}\right) .
$$

This completes the proof of Theorem 1 .

\section{Inequalities}

Theorem 1 is now applied to obtain sharp estimates for the vertex number of the $L_{j}$ weighted typical $k$-face $Z_{k, j}$. The set of all $\left(H_{1}, \ldots, H_{d-j}\right) \in G(d, d-1)^{d-j}$ that are not in general position has $\phi_{d-j}$-measure zero, since $\nabla_{d-j}\left(u_{1}, \ldots, u_{d-j}\right)=0$ if 
$u_{1}, \ldots, u_{d-j}$ are linearly dependent. We use Theorem 1 with $h(P):=f_{0}(P)$, and hence with

$$
h_{k, j}\left(\widehat{X} \cup\left\{H_{1}, \ldots, H_{d-j}\right\}, 0\right)=\sum_{i=1}^{n} f_{0}\left(P_{i}\right),
$$

where $P_{1}, \ldots, P_{n}$ are the $k$-faces of the tessellation induced by $\widehat{X} \cup\left\{H_{1}, \ldots, H_{d-j}\right\}$ that contain 0 . Each of the vertices counted in (7) is generated by $r$ hyperplanes from $H_{1}, \ldots, H_{d-j}$ and $d-r$ facets of the zero cell $Z_{0}$ of $X$, for some $r \in\{d-k, \ldots$, $d-j\}$. Every such vertex belongs to exactly $2^{k-(d-r)}\left(\begin{array}{c}r \\ d-k\end{array}\right)$ of the $k$-faces $P_{1}, \ldots, P_{n}$. This gives

$$
\begin{aligned}
& h_{k, j}\left(\widehat{X} \cup\left\{H_{1}, \ldots, H_{d-j}\right\}, 0\right) \\
& \quad=2^{k-d} \sum_{r=d-k}^{d-j} 2^{r}\left(\begin{array}{c}
r \\
d-k
\end{array}\right) \sum_{1 \leq i_{1}<\cdots<i_{r} \leq d-j} f_{0}\left(Z_{0} \cap H_{i_{1}} \cap \cdots \cap H_{i_{r}}\right) .
\end{aligned}
$$

We can now argue as in [8] and make use of the associated zonoid and the inequalities for the volume product of a zonoid (that is, apply [9, (10.52)] to the section process $\widehat{X} \cap H_{i_{1}} \cap \cdots \cap H_{i_{r}}$ ) and obtain the inequalities

$$
2^{d-r} \leq \mathbb{E} f_{0}\left(Z_{0} \cap H_{i_{1}} \cap \cdots \cap H_{i_{r}}\right) \leq 2^{r-d}(d-r) ! \kappa_{d-r}^{2},
$$

where $\kappa_{m}$ denotes the volume of the $m$-dimensional unit ball. This gives

$$
\begin{aligned}
& \frac{2^{j-k}}{\left(\begin{array}{c}
d-j \\
d-k
\end{array}\right)} \mathbb{E} h_{k, j}\left(\widehat{X} \cup\left\{H_{1}, \ldots, H_{d-j}\right\}, 0\right) \\
& \quad \leq \frac{2^{j-k}}{\left(\begin{array}{c}
d-j \\
d-k
\end{array}\right)} 2^{k-d} \sum_{r=d-k}^{d-j} 2^{r}\left(\begin{array}{c}
r \\
d-k
\end{array}\right)\left(\begin{array}{c}
d-j \\
r
\end{array}\right) \cdot 2^{r-d}(d-r) ! \kappa_{d-r}^{2} \\
& \quad=2^{j-2 k} \sum_{i=0}^{k-j} 2^{2 i}\left(\begin{array}{c}
k-j \\
i
\end{array}\right)(k-i) ! \kappa_{k-i}^{2} .
\end{aligned}
$$

Similarly, the lower bound $2^{k}$ is obtained, and integration with the probability measure $\phi_{d-j}$ gives the final result.

Theorem 2 For $0 \leq j \leq k \leq d$,

$$
2^{k} \leq \mathbb{E} f_{0}\left(Z_{k, j}\right) \leq 2^{j-2 k} \sum_{i=0}^{k-j} 2^{2 i}\left(\begin{array}{c}
k-j \\
i
\end{array}\right)(k-i) ! \kappa_{k-i}^{2} .
$$

If $X$ is isotropic, then equality holds on the right side. For $k \geq 2$, equality on the left side holds if and only if $X$ is a parallel mosaic.

The discussion of the equality cases is the same as in [8]. 
In the isotropic case, we list the values for $k=2$ :

$$
\begin{aligned}
& \mathbb{E} f_{0}\left(Z_{2,0}\right)=\frac{\pi^{2}}{8}+3 \approx 4.23, \\
& \mathbb{E} f_{0}\left(Z_{2,1}\right)=\frac{\pi^{2}}{4}+2 \approx 4.47, \\
& \mathbb{E} f_{0}\left(Z_{2,2}\right)=\frac{\pi^{2}}{2} \approx 4.93 .
\end{aligned}
$$

Thus, weighting by the vertex number gives the smallest value for the expected number of vertices, which may seem paradoxical at first sight.

\section{References}

1. Favis, W., Weiss, V.: Mean values of weighted cells of stationary Poisson hyperplane tessellations of $R^{d}$. Math. Nachr. 93, 37-48 (1998)

2. Matheron, G.: Ensembles aléatoires, ensembles semi-markoviens et polyèdres poissoniens. Adv. Appl. Probab. 4, 508-541 (1972)

3. Matheron, G.: Hyperplans Poissoniens et compact de Steiner. Adv. Appl. Probab. 6, 563-579 (1974)

4. Matheron, G.: Random Sets and Integral Geometry. Wiley, New York (1975)

5. Miles, R.E.: Random polytopes: the generalisation to $n$ dimensions of the intervals of a Poisson process. Ph.D. thesis, Cambridge University (1961)

6. Miles, R.E.: Poisson flats in Euclidean spaces. II: Homogeneous Poisson flats and the complementary theorem. Adv. Appl. Probab. 3, 1-43 (1971)

7. Miles, R.E.: A synopsis of 'Poisson flats in Euclidean spaces'. Izv. Akad. Nauk Arm. SSR, Mat. 5, 263-285 (1970). Reprinted in: Harding, E.F., Kendall, D.G. (eds.) Stochastic Geometry, pp. 202-227. Wiley, New York (1974)

8. Schneider, R.: Weighted faces of Poisson hyperplane tessellations. Adv. Appl. Prob. (SGSA) 41, 682694 (2009)

9. Schneider, R., Weil, W.: Stochastic and Integral Geometry. Springer, Berlin Heidelberg (2008) 